specific standardised tests in order to achieve effective neurodevelopmental surveillance of this vulnerable population.

\section{G111 IMPACT OF TRANSCUTANEOUS BILLIRUBINOMETER TESTING ON BABIES WITH VISIBLE JAUNDICE BY COMMUNITY MIDWIVES ON HOSPITAL REFERRALS}

${ }^{1} A$ Ramachandran, ${ }^{2} \mathrm{~L}$ Owen, ${ }^{1} \mathrm{R}$ Morris. 'Singleton Hospital, ABM University Health Board, Swansea, UK: ${ }^{2}$ Neath Hospital, ABMU Health Board, Swansea, UK

\subsection{6/archdischild-2015-308599.110}

Introduction Referrals of newborn babies with jaundice to hospital has emotional and financial impact on parents and increases work load of hospitals. We report results from a service evaluation project looking at the introduction of transcutaneous bilirubinometers (TsB) testing to community midwives using JM $103 \mathrm{TsB}$ as recommended in NICE neonatal jaundice guideline (CG 98).

Aim Assess impact of TsB to reduce avoidable hospital referral by community midwifes.

Methodology A limited pilot project undertaken in 2013 showed that in the absence of TsB only $40 \%$ of babies referred to hospital for neonatal jaundice were admitted. Average waiting time before medical review was $255 \mathrm{~min}$ in paediatric assessment units. With innovative project funding from the Welsh Government the project team trained and equipped all 80 community midwives in the Health Board with a TsB each from February 2014. A clear referral pathway was established and activity recorded.

Results Data was collected on all babies reviewed by midwives at home for a 6 month period between 01/02/2014 to 31/07/ 2014 in the Health Board. Compliance of midwifes to protocol was high. 5647 babies were reviewed by midwives, 1046 (19\%) were tested with TsB for visible jaundice and 63 (1\%) were referred to hospital. Of 48 babies audited $69 \%$ of those referred were admitted and 54\% received phototherapy. Average waiting time reduced to a quarter at $58 \mathrm{~min}$. Service was given a maximum satisfaction rating by most parents. JM 103 TsB was found to be $100 \%$ specific in predicting need for hospital admission (95\% CI 78\%-100\%) and need for phototherapy (95\% CI 84.4 $-100 \%)$.

Conclusion Judiciously used TsB can half avoidable hospital referrals and quarter waiting times for babies with neonatal jaundice in first 2 weeks of life. This combined with immediate reassurance and a non invasive pain free test resulted in high parent satisfaction scores. Financial benefits incur to parents from reduced travel costs and to hospitals from reduced blood testing and saved medical time.

\section{G112 CHORIOAMNIONITIS IN PRETERM INFANTS IS NOT ASSOCIATED WITH BRAIN SIZE AND MATURATION AT TERM EQUIVALENT AGE}

${ }^{1} \mathrm{CL}$ Granger, ${ }^{2} \mathrm{~A}$ Spittle, ${ }^{3,4} \mathrm{LW}$ Doyle, ${ }^{4} \mathrm{~J}$ Pyman, ${ }^{3,5} \mathrm{JM}$ Walsh, ${ }^{3,5} \mathrm{JLY}$ Cheong. ${ }^{1}$ Neonatal Intensive Care Unit, Royal Hospital for Sick Children, Glasgow, UK; ${ }^{2}$ Murdoch Children's Research Institute, University of Melbourne, Melbourne, Australia; ${ }^{3}$ Newborn Research Department, The Royal Women's Hospital, Melbourne, Australia; ${ }^{4}$ Anatomical Pathology, The Royal Women's Hospital, Melbourne, Australia; ${ }^{5}$ Neonatal Intensive Care Unit, The Royal Women's Hospital, Melbourne, Australia

\subsection{6/archdischild-2015-308599.111}

Introduction Chorioamnionitis is a risk factor for adverse neurodevelopment in preterm infants but the effect on brain size or maturation is unclear. This study aimed to determine the association between perinatal characteristics and histological chorioamnionitis in a cohort of preterm infants. We also aimed to establish the association between chorioamnionitis and brain size and maturation.

Methods A large prospective cohort of preterm infants was identified and data from placental histology reports were

Abstract G112 Table 1 Participant characteristics

\begin{tabular}{|c|c|c|c|c|}
\hline & \multicolumn{3}{|c|}{ Chorioamnionitis } & \multirow[t]{2}{*}{ P value } \\
\hline & $\begin{array}{c}\text { None } \\
(n=179)\end{array}$ & $\begin{array}{l}\text { Maternal response only } \\
\qquad(n=19)\end{array}$ & $\begin{array}{l}\text { Both maternal and fetal } \\
\text { response } \\
(n=39)\end{array}$ & \\
\hline Antenatal steroids & $136(76.0)$ & $17(89.5)$ & $32(82.1)$ & 0.32 \\
\hline $\begin{array}{l}\text { Gestation at birth in weeks, } \\
\text { mean }(\mathrm{SD})^{*}\end{array}$ & $31.8(3.4)$ & $30.0(3.7)$ & $29.1(3.6)$ & $<0.0001$ \\
\hline $\begin{array}{l}\text { Gestational age groups } \\
\text { - } \quad \text { Very preterm } \\
\text { Mod/late preterm }\end{array}$ & $\begin{array}{c}71(39.7) \\
108(60.3)\end{array}$ & $\begin{array}{c}13(68.4) \\
6(31.6)\end{array}$ & $\begin{array}{l}28(71.8) \\
11(28.3)\end{array}$ & $<0.0001$ \\
\hline $\begin{array}{l}\text { Birthweight in grams, mean } \\
\text { (SD)* }\end{array}$ & $1619(613)$ & $1597(928)$ & $1331(559)$ & 0.04 \\
\hline $\begin{array}{l}\text { Birthweight z-score, mean } \\
(\mathrm{SD})^{*}\end{array}$ & $-0.7(1.1)$ & $0.1(1.1)$ & $-0.2(0.7)$ & 0.0003 \\
\hline Small for gestational age & $30(16.8)$ & $0(0)$ & $0(0)$ & - \\
\hline Male & $91(50.8)$ & $11(57.9)$ & $18(46.2)$ & 0.70 \\
\hline Multiple birth & $87(48.6)$ & $3(15.8)$ & $9(23.1)$ & 0.001 \\
\hline $\begin{array}{l}\text { Ruptured membranes }>24 \\
\text { hours }\end{array}$ & $16(9.3)$ & $5(26.3)$ & $22(56.4)$ & $<0.0001$ \\
\hline Any IVH & $10(5.6)$ & $3(15.8)$ & $10(25.6)$ & $<0.0001$ \\
\hline Cystic PVL & $0(0)$ & $1(5.3)$ & $0(0)$ & - \\
\hline Chronic lung disease & $23(12.9)$ & $3(15.8)$ & $10(25.6)$ & 0.13 \\
\hline Postnatal steroids & $11(7.9)$ & $1(5.9)$ & $3(9.4)$ & 0.87 \\
\hline
\end{tabular}

Data are $\mathrm{n}(\%)$ unless otherwise specified; SD-standard deviation, IVH - intraventricular haemorrhage, PVL- periventricular leukomalacia; All comparisons using chi-squared except for those marked * where ANOVAused instead 
Abstract G112 Table 2 Association between chorioamnionitis and MRI measures

\begin{tabular}{|c|c|c|c|c|c|}
\hline & \multicolumn{3}{|c|}{ Chorioamnionitis } & \multicolumn{2}{|c|}{ Coefficient $(95 \% \mathrm{Cl})$ Adjusted p value ${ }^{\Omega}$} \\
\hline & $\begin{array}{l}\text { None } \\
(n=165)\end{array}$ & $\begin{array}{c}\text { Maternal response } \\
\text { only } \\
(n=15)\end{array}$ & $\begin{array}{l}\text { Both maternal and } \\
\text { fetal response } \\
\text { ( } n=32)\end{array}$ & $\begin{array}{l}\text { Maternal response compared with } \\
\text { none }\end{array}$ & $\begin{array}{l}\text { Both maternal \& fetal response } \\
\text { compared with none }\end{array}$ \\
\hline \multicolumn{6}{|l|}{ Brain measures } \\
\hline $\begin{array}{l}\text { Brain biparietal diameter } \\
(\mathrm{mm})\end{array}$ & $82.5(4.5)$ & $84.0(3.8)$ & $83.1(4.2)$ & $\begin{array}{c}2.0(-0.1,3.9) \\
p=0.05\end{array}$ & $\begin{array}{c}1.6(-0.1,3.2) \\
p=0.06\end{array}$ \\
\hline $\begin{array}{l}\text { Corpus callosum - genu } \\
(\mathrm{mm})\end{array}$ & $4.3(1.4)$ & $3.6(1.6)$ & $4.1(1.4)$ & $\begin{array}{c}-0.2(-0.8,0.3) \\
p=0.42\end{array}$ & $\begin{array}{c}0.3(-0.1,0.8) \\
p=0.14\end{array}$ \\
\hline $\begin{array}{l}\text { Corpus callosum - body } \\
\text { (mm) }\end{array}$ & $2.1(0.5)$ & $2.0(0.6)$ & $2.0(0.6)$ & $\begin{array}{c}0.02(-0.2,0.3) \\
p=0.88\end{array}$ & $\begin{array}{c}0.08(-0.1,0.3) \\
p=0.48\end{array}$ \\
\hline $\begin{array}{l}\text { Corpus callosum - } \\
\text { splenium (mm) }\end{array}$ & $3.8(1.0)$ & $3.5(1.6)$ & $3.4(1.0)$ & $\begin{array}{c}-0.03(-0.7,0.6) \\
p=0.92\end{array}$ & $\begin{array}{c}-0.05(-0.4,0.3) \\
p=0.79\end{array}$ \\
\hline $\begin{array}{l}\text { Deep nuclear gray matter } \\
\left(\mathrm{mm}^{2}\right)\end{array}$ & $1235.6(106.2)$ & $1262.9(102.5)$ & $1244.6(105.9)$ & $\begin{array}{c}23.5(-28.5,75.6) \\
p=0.38\end{array}$ & $\begin{array}{c}13.2(-28.9,55.3) \\
p=0.54\end{array}$ \\
\hline $\begin{array}{l}\text { Transcerebellar diameter } \\
(\mathrm{mm})\end{array}$ & $55.5(3.1)$ & $54.8(2.8)$ & $55.2(3.0)$ & $\begin{array}{c}-0.4(-1.7,1.0) \\
p=0.58\end{array}$ & $\begin{array}{c}0.6(-0.4,1.5) \\
p=0.22\end{array}$ \\
\hline \multicolumn{6}{|l|}{ CSF measures } \\
\hline $\begin{array}{l}\text { Right lateral ventricle } \\
(\mathrm{mm})\end{array}$ & $5.9(1.8)$ & $6.7(3.5)$ & $6.2(1.9)$ & $\begin{array}{c}0.7(-1.0,2.3) \\
p=0.45\end{array}$ & $\begin{array}{c}0.2(-0.5,0.8) \\
p=0.63\end{array}$ \\
\hline Left lateral ventricle $(\mathrm{mm})$ & $5.6(1.8)$ & $6.1(3.1)$ & $5.8(2.0)$ & $\begin{array}{c}0.3(-1.1,1.8) \\
p=0.64\end{array}$ & $\begin{array}{c}-0.1(-0.8,0.6) \\
p=0.77\end{array}$ \\
\hline $\begin{array}{l}\text { Interhemispheric distance } \\
(\mathrm{mm})\end{array}$ & $2.8(1.3)$ & $2.9(1.7)$ & $3.2(1.9)$ & $\begin{array}{c}-0.1(-0.9,0.8) \\
p=0.83\end{array}$ & $\begin{array}{c}0.03(-0.7,0.7) \\
p=0.93\end{array}$ \\
\hline \multicolumn{6}{|l|}{ Brain maturation } \\
\hline $\begin{array}{l}\text { PLIC myelination } \\
\text { complete, } \mathrm{n}(\%) \\
\end{array}$ & $139(84.2)$ & $14(93.3)$ & $27(84.4)$ & $\begin{array}{c}2.0(0.2,23.6)^{\bullet} \\
p=0.59\end{array}$ & $\begin{array}{c}0.9(0.3,2.6)^{*} \\
p=0.89\end{array}$ \\
\hline $\begin{array}{l}\text { Gyral maturation }>38 \\
\text { weeks, } \mathrm{n}(\%)\end{array}$ & $94(57.0)$ & $5(33.3)$ & $13(40.6)$ & $\begin{array}{c}0.4(0.2,1.3)^{*} \\
p=0.13\end{array}$ & $\begin{array}{c}1.0(0.4,2.4)^{*} \\
p=0.92\end{array}$ \\
\hline
\end{tabular}

* Results presented as odds ratios $(95 \% \mathrm{Cl})$

\& Using GEE with robust standard errors to account for multiple births, all analyses adjusted for gestation at MRI, gestation at birth, sex and bw sds

collected and chorioamnionitis, characterised into either no inflammatory response, maternal response only or both fetal and maternal response. All infants had brain MRI scans at term equivalent age. T1 and T2 weighted images were systematically scored for size of cerebral structures, degree of myelination, gyral maturation, signal abnormalities and presence of cysts. Differences between groups were compared using linear regression for continuous variables and logistic regression for categorical variables.

Results Of the 237 infants included in this study, 58 (24.5\%) had histological evidence of chorioamnionitis.

Table 1 summarises the demographic data. Infants with evidence of chorioamnionitis on placental histology had higher rates of preterm delivery $(\mathrm{p}<0.0001)$, low birth weight $(\mathrm{p}=$ $0.04)$ and prolonged rupture of membranes $(p<0.0001)$. Those with chorioamnionitis had higher rates of intraventricular haemorrhage ( $\mathrm{p}<0.0001)$.

Chorioamnionitis, however, was not associated with brain injury or delayed maturation on qualitative MRI assessment. The MRI scoring data is shown in Table 2.

\section{G113(P) SURVIVAL IN INFANTS BORN AT LESS THAN 24 WEEKS GESTATION - A POPULATION BASED STUDY}

${ }^{1} S$ Tiwary, ${ }^{2} \mathrm{R}$ Geethanath, ${ }^{3} \mathrm{~B}$ Reichert, ${ }^{3} \mathrm{~S}$ Janakiraman, ${ }^{4} \mathrm{~S}$ Garg, ${ }^{1} \mathrm{~N}$ Embleton. ${ }^{1}$ Newcastle Neonatal Service, Newcastle Hospitals NHS Foundation Trusts, Newcastle-Upon-Tyne, UK; ${ }^{2}$ Neonatal Unit, Sunderland Royal Infirmary, Sunderland, UK; ${ }^{3}$ Neonatal Unit, University Hospital of North Tees, Stockton-Upon-Tees, UK; ${ }^{4}$ Neonatal Unit, James Cook University Hospital, Middlesborough, UK

\subsection{6/archdischild-2015-308599.112}

Background The management of infants born at less than 24 weeks gestation $(<24 \mathrm{w})$ remains contentious. In a large population based cohort 1993-2008 we previously showed no appreciable increase in long-term survival for liveborn infants $<24 \mathrm{w}$, although median age at death increased over each successive 5 -year epochs. We aimed to review recent changes in survival $<24 \mathrm{w}$, and to determine risk factors that might predict non-survival at an earlier age.

Methods Cases (live-born infants at 22-23w) were identified using a well-validated population based database (Regional Maternity Survey Office, Northern Region, UK) for the 5-year epoch 2008-2012. We included cases still alive at $6 \mathrm{~h}$ of age, in order to identify a group where the intention to provide active management appears likely. Survivors were identified from unit databases of the 4 tertiary level neonatal units, and individual case notes reviewed.

Results During the study period 55 infants $(56 \%$ male) born at $22-23 \mathrm{w}$ ( $\mathrm{n}=2$ at $22 \mathrm{w}$ ) were alive at $6 \mathrm{~h}$ (birth prevalence $\sim 4: 10,000)$. Of these $24 / 55$ infants $(44 \%)$ were alive at 12 months age $(50 \%$ male); $18 / 24$ survivors received at least one dose of steroids. Median survival of non-survivors was 2.65 days (excludes one case known to have died at $>2$ years age). $7 / 31$ non-survivors had a laparotomy, 3 had retinopathy of prematurity (ROP) treatment, 3 had PDA ligation, and only 2/31 ever achieved full feeds. Amongst survivors, 20/24 required some form of invasive surgery/intervention (4 laparotomy, 2 other surgery, 15 laser ROP, 2 Avastin for ROP, and 14 PDA ligation.) There was no significant difference in birthweight between survivors and non-survivors. Initial analysis did not identify early postnatal factors that enabled clear identification of infants who subsequently died.

Conclusion Survival at $<24 \mathrm{w}$ has increased dramatically in this population over the last few years, but was not associated with a prolonged period of intensive care in those who did not survive. However, there was considerable morbidity (e.g. surgical procedures) in both groups. Increased survival may be due to several factors, and will be affected by clinician and parental attitudes. 